\title{
The Effect of Bolus Taste Variability on Swallowing in Normal Subjects Versus Stroke Patients
}

\author{
Original \\ Article \\ Alzahra Omara ${ }^{1}$, Omayma Afsah ${ }^{2}$, Mohammed Darwish ${ }^{1}$ Ibrahim Elmenshawi', \\ Tamer Abou-Elsaad \\ ${ }^{1}$ Phoniatric Unit, Faculty of Medicine, Tanta University, Tanta, ${ }^{2}$ Phoniatric Unit, Department \\ of ORL, ${ }^{3}$ Department of Neurology, Faculty of Medicine, Mansoura University, Mansoura, \\ Egypt.
}

\begin{abstract}
Objective: The objective of this study was to assess the effect of bolus taste variability on oropharyngeal swallowing in normal individuals versus stroke patients to explore its role in the management of oropharyngeal dysphagia.

Patients and Methods: This study was conducted on 30 patients with oropharyngeal dysphagia due to stroke and 30 healthy adults, who were assessed during swallowing of $5 \mathrm{ml}$ liquid boluses and $5 \mathrm{ml}$ semisolid boluses of different tastes: sweet, salty, sour, and bitter/spicy. They were examined using videofluoroscopy and nasopharyngeal videofibroscopy to analyze temporal measures of swallowing, penetration aspiration scale, and Mansoura FEES Residue Rating Scale.

Results: In all bolus tastes, both fluids and semisolid consistencies demonstrated significantly longer temporal measures in the patient group than in the control group. All temporal measures were consistently longer in the semisolid consistency than in the fluid consistency. However, this difference did not reach statistical significance in some cases (e.g., sweet taste in the patient group and sour taste in the control group). Non-significant differences were found between different tastes as regards their effects on PAS scores as well as on residue scale scores.

Conclusion: Different tastes have different temporal measures, with the sweet taste being the shortest and bitter taste being the most prolonged durations in both normal and stroke patients. Neither studied taste correlated significantly with penetration /aspiration of food boluses nor with their residue scale. The food taste management option could be used in different physiological breakdowns, which necessitates further research.
\end{abstract}

Key Words: Dysphagia, sour, stroke, sweet, taste.

Received: 6 November 2020, Accepted: 7 January 2021

Corresponding Author: Omayma Afsah, MD, Phoniatric unit, ORL Department, Faculty of Medicine, Mansoura University, Mansoura, 35516, Egypt, Tel.: 01002929720,E-mail: omafsah@yahoo.com

ISSN: 2090-0740, 2021, Vol.22

\section{INTRODUCTION}

Dysphagia has a significant impact on the quality of life of stroke survivors ${ }^{[1]}$. It may result in malnutrition, dehydration, respiratory infections, and pneumonia ${ }^{[2]}$. Aspiration pneumonia remains the third principal cause of death during the first month after stroke onset and accounts for approximately $34 \%$ of all stroke deaths afterwards. Increased severity of aspiration was associated with a higher incidence of pneumonia, especially in immunocompromised stroke patients with poor oral health $^{[3]}$.

Taste potentially affects swallowing as it stimulates swallowing coordination and autonomic nervous system responses $^{[4]}$. Sensory input is essential to initiate and regulate swallowing, and the relation of oral sensory pathways to swallow motor dysfunctions has been established $^{[5]}$.
Taste disorders after stroke are frequent and may not be detected by the physicians and patients ${ }^{[6]}$. Loss of capacity to distinguish salty, sweet, or sour tastes has been reported, and, in the majority of cases, these functional disorders continue for more than 18 months $^{[7]}$.

Although swallowing difficulties may get better naturally in about $10 \%$ of stroke patients, problems can last for more than six months ${ }^{[8]}$. It has been proposed that bolus taste can modify swallowing in stroke pa $\neg$ tients $[9,10]$ and may have a role in managing patients with dysphagia after stroke $^{[11]}$.

Both modified barium swallow (MBS) and Fiberoptic endoscopic evaluation of swallowing (FEES) are commonly used in dysphagia evaluation. They have been compared for clinical indications, outcomes ${ }^{[12]}, \operatorname{cost}^{[13]}$, the ability to detect aspiration, and scoring several parameters, including pharyngeal clearance ${ }^{[14,15]}$. 
The effect of taste on the physiology of swallowing remains equivocal. This may be due to differences in methods employed or the type of stimulus presented poses a challenge ${ }^{[16]}$. Taste induces different autonomic nervous system responses, with sweet taste causing the weakest response while the unpleasant tastes (salty, sour, and bitter) making more robust responses, with bitter taste producing the strongest ones ${ }^{[17]}$.

This study's objective was to assess the effect of bolus taste variability on oropharyngeal swallowing in normal individuals versus stroke patients to explore its role in managing oropharyngeal dysphagia.

\section{PATIENTS AND METHODS:}

\subsection{Subjects:}

This prospective cross-sectional study was carried out at the Unit of Phoniatrics, Mansoura University Hospital from July 2018 to March 2019. The study included 60 adults in the age range of 30-75 years who were divided into two groups. Group I (patient group) consisted of 30 patients with oropharyngeal dysphagia due to stroke. We included patients who understood and followed verbal instructions who suffered from a stroke, whatever its type (ischemic or hemorrhagic) or its site who proved to be safe to receive oral feedings when examined by FEES. Severely compromised patients and patients with severe cogni $\neg$ tive impairment and those with other neurological etiologies for their dysphagia were excluded.

Group II (control group) consisted of 30 healthy adults with no history of any neurological or gastroesophageal diseases and no history of medical or surgical etiology that could impair swallowing function.

\subsection{Methods:}

Group I (patient group) were initially subjected to the following protocol of assessment: patient's interview, auditory perceptual assessment (APA) of language, speech and voice, and clinical examination including general examination, preliminary visualization of oropharyngo-laryngeal tract, neck examination, neurological examination as well as observations during trial feeding.

Each subject was assessed during swallowing of 5 $\mathrm{ml}$ liquid boluses and $5 \mathrm{ml}$ semisolid boluses of different tastes, and each taste/consistency was examined twice. Lemon juice, sucrose, saline, tea, and a mix of grinded dry red chili and grinded black pepper were used to represent sour, sweet, salty, bitter, and spicy tastes, respectively.

Liquid boluses of sour taste $(50 \mathrm{ml}$ of water with $20 \mathrm{ml}$ of lemon juice), sweet taste ( $50 \mathrm{ml}$ of water with $10 \mathrm{gm}$ of sucrose), salty taste (saline $0.9 \mathrm{NaCl})$, bitter taste $(50 \mathrm{ml}$ of water with $5 \mathrm{gm}$ of tea) were prepared.
For preparing the semisolid boluses, $50 \mathrm{gm}$ of starch were dissolved in $150 \mathrm{ml}$ boiled water with the addition of (20 ml lemon juice) for sour taste, the addition of (15 gm sucrose) for sweet taste, addition of (10 $\mathrm{ml}$ saline $\mathrm{NaCl})$ for salty taste, and addition of a mix of grinded dry red chili and grinded black pepper for a spicy taste. These consistencies were placed in a clean container on fire till forming semisolid consistencies, then allowed to cool and put in clean plastic containers. Barium was added (about $10 \mathrm{gm}$ ) to all samples, and all boluses were offered at room temperature.

\subsubsection{Videofluoroscopic swallowing examination:}

All participants were examined using the MBS procedure with videofluoroscopy at the radiology department, Mansoura University Hospitals. Digital recordings were made for each bolus. Each study subject was given the $5 \mathrm{ml}$ bolus via spoon and instructed to keep the bolus in the mouth then swallow when the command swallow was given. Between recording sequences, subjects were given a spoon of unlabeled water to wash out any residue from a previous swallow. The videofluoroscopic studies were conducted in the lateral plane according to the procedure outlined by Logemann ${ }^{[18]}$.

Video records were analyzed for the following temporal measures of oropharyngeal swallowing:

1) Oral transit duration (OTD): from the initiation of posterior bolus movement to arrival of bolus head at the ramus of mandible.

2) Oral clearance duration (OCD): from the initiation of posterior bolus movement to arrival of bolus tail at the ramus of mandible.

3) Pharyngeal transit duration (PTD): from the arrival of bolus head at the ramus of mandible to bolus head enter upper esophageal sphincter.

4) Pharyngeal clearance duration (PCD): from the arrival of bolus head at the ramus of mandible to bolus tail enter upper esophageal sphincter.

5) Hyoid maximal elevation duration (HMED): from hyoid first maximum elevation to hyoid last maximum elevation

6) Total swallow duration (TSD): summing both oral transit and pharyngeal clearance duration.

Additionally, video records were analyzed for penetration or aspiration based on the penetration aspiration scale developed by Rosenbek et al ${ }^{[19]}$ (Appendix A) 


\subsubsection{Flexible endoscopic evaluation of swallowing:}

All participants were examined using nasopharyngeal videofibroscopy connected to a video camera and laptop with a TV tuner. The fiberscope was passed through the nasal cavity to the level just above the epiglottis (home position) with a view of the tongue's base, the entire hypopharynx, and larynx. The supralaryngeal region was observed and digitally recorded while swallowing the same tastes and consistencies previously examined with Videofluoroscopy.

Video records were analyzed for determination and scoring of residue based on the Mansoura FEES Residue Rating scale (MFRRS) developed by Sabry, Coyle and Abou-Elsaad ${ }^{[20]}$ (appendix B).

\subsubsection{Statistical analysis:}

Data were analyzed using SPSS (statistical package for social science) version 21. Qualitative data were expressed as number and percentage. Quantitative data were expressed as mean and standard deviation. One-way ANOVA was used to compare more than two materials, followed by the Bonferroni test to compare between every two measures. The student t-test was used to compare two groups. Results were considered significant when the probability of error was less than or equal to $5 \%(p \leq 0.05)$.

\subsubsection{Ethical consideration:}

The Institutional Review Board of Mansoura Faculty of Medicine approved the study (MD/17.01.49c). Written informed consent was obtained from all subjects or their guardians before the study.

\section{RESULTS:}

\subsection{Demographic data:}

This study was conducted on 60 adults ( 37 males and 23 females) in the age range of 30-75 years (mean $55 \pm$ 8.65 ) divided into two groups.
Group I (patient group) included 30 patients with oropharyngeal dysphagia due to stroke who presented from day 1 to 21 days after the stroke onset (mean $8.9 \pm$ 4.5). They included ( 19 males, 11 females) in the age range 45-75 (mean $63.2 \pm 9.79$ ) years. In most patients $(46.7 \%$ ), the etiology of oropharyngeal dysphagia was left cortical stroke. Group II (control group) consisted of 30 healthy volunteers (18 males and 12 females) in the age range of $30-50$ years (mean $35.8 \pm 7.58$ ). Both groups were matched for sex.

\subsection{Results of the videofluoroscopic assessment of} swallowing:

The videofluoroscopic assessment included an assessment of temporal measures of swallowing and analysis of penetration aspiration scale.

\subsubsection{Effects of individual bolus tastes on temporal}

\section{measures of swallowing:}

In all bolus tastes, both fluids and semisolid consistencies demonstrated significantly longer temporal measures in the patient group than in the control group, except hyoid maximal elevation duration (HMED) in nearly all tastes. The within-group comparison revealed the differential effect of bolus consistency on both patient and control groups' temporal measures. All temporal measures were consistently longer in the semisolid consistency than in the fluid consistency. However, this difference did not reach statistical significance in some cases (e.g., sweet taste in the patient group and sour taste in the control group), as shown in Tables (1-4).

Table 1: Effect of sweet taste on temporal measures of swallowing:

\begin{tabular}{|c|c|c|c|c|}
\hline $\begin{array}{l}\text { Temporal measures of } \\
\text { sweet taste in msecs }\end{array}$ & Consistency & Group I $(n=30)$ & Group II $(\mathrm{n}=30)$ & $\mathrm{P} 2$ \\
\hline \multirow{3}{*}{ OTD } & Fluids (Mean \pm SD) & $0.95 \pm 0.46$ & $0.26 \pm 0.10$ & $0.001^{*}$ \\
\hline & Semisolids (Mean \pm SD) & $1.45 \pm 0.80$ & $0.47 \pm 0.18$ & $0.001^{*}$ \\
\hline & $\mathrm{P} 1$ & 0.356 & $0.001^{*}$ & \\
\hline \multirow{3}{*}{ PTD } & Fluids (Mean \pm SD) & $1.61 \pm 1.04$ & $0.35 \pm 0.12$ & $0.001^{*}$ \\
\hline & Semisolids (Mean \pm SD) & $2.37 \pm 3.12$ & $0.42 \pm 0.14$ & $0.001^{*}$ \\
\hline & $\mathrm{P} 1$ & $0.039^{*}$ & 0.589 & \\
\hline \multirow{3}{*}{ OCD } & Fluids (Mean \pm SD) & $1.70 \pm 0.75$ & $0.50 \pm 0.16$ & $0.001^{*}$ \\
\hline & Semisolids (Mean \pm SD) & $2.75 \pm 2.33$ & $0.70 \pm 0.18$ & $0.001^{*}$ \\
\hline & $\mathrm{P} 1$ & 0.497 & $0.001^{*}$ & \\
\hline
\end{tabular}




\begin{tabular}{|c|c|c|c|c|}
\hline \multirow{3}{*}{ PCD } & Fluids (Mean \pm SD) & $2.12 \pm 0.92$ & $0.65 \pm 0.11$ & $0.001^{*}$ \\
\hline & Semisolids (Mean \pm SD) & $3.26 \pm 3.24$ & $0.80 \pm 0.27$ & $0.001^{*}$ \\
\hline & $\mathrm{P} 1$ & 0.409 & $0.005^{*}$ & \\
\hline \multirow{3}{*}{ TSD } & Fluids (Mean \pm SD) & $3.07 \pm 1.13$ & $0.91 \pm 0.17$ & $0.001^{*}$ \\
\hline & Semisolids (Mean \pm SD) & $4.86 \pm 3.89$ & $1.28 \pm 0.32$ & $0.001^{*}$ \\
\hline & $\mathrm{P} 1$ & 0.319 & $0.001^{*}$ & \\
\hline \multirow{3}{*}{ HMED } & Fluids (Mean \pm SD) & $0.35 \pm 0.09$ & $0.29 \pm 0.10$ & $0.001^{*}$ \\
\hline & Semisolids (Mean \pm SD) & $0.45 \pm 0.14$ & $0.41 \pm 0.20$ & 0.329 \\
\hline & $\mathrm{P} 1$ & 0.230 & $0.004^{*}$ & \\
\hline
\end{tabular}

Data are expressed as mean and standard deviation.

Student $\mathrm{t}$ test was used to compare between both groups.

$P$ value $<0.05$ is significant, $P$ value $>0.05$ non-significant.

$\mathrm{P} 1$ represent within group comparison.

$\mathrm{P} 2$ represent between group comparison.

Table 2: Effect of sour taste on temporal measures of swallowing:

\begin{tabular}{|c|c|c|c|c|}
\hline $\begin{array}{l}\text { Temporal measures of } \\
\text { sour taste in msecs }\end{array}$ & Consistency & Group I $(n=30)$ & Group II $(\mathrm{n}=30)$ & $\mathrm{P} 2$ \\
\hline \multirow{3}{*}{ OTD } & Fluids (Mean \pm SD) & $1.10 \pm 0.56$ & $0.38 \pm 0.15$ & $0.001^{*}$ \\
\hline & Semisolids (Mean \pm SD) & $1.65 \pm 0.86$ & $0.39 \pm 0.14$ & $0.001^{*}$ \\
\hline & $\mathrm{P} 1$ & $0.005^{*}$ & 0.745 & \\
\hline \multirow{3}{*}{ PTD } & Fluids (Mean \pm SD) & $1.75 \pm 1.39$ & $0.41 \pm 0.18$ & $0.001^{*}$ \\
\hline & Semisolids (Mean \pm SD) & $1.93 \pm 1.70$ & $0.50 \pm 0.25$ & $0.001^{*}$ \\
\hline & $\mathrm{P} 1$ & 0.664 & 0.084 & \\
\hline \multirow{3}{*}{ OCD } & Fluids (Mean \pm SD) & $1.81 \pm 1.07$ & $0.63 \pm 0.26$ & $0.001^{*}$ \\
\hline & Semisolids (Mean \pm SD) & $2.69 \pm 1.73$ & $0.67 \pm 0.26$ & $0.001^{*}$ \\
\hline & $\mathrm{P} 1$ & $0.022^{*}$ & 0.537 & \\
\hline \multirow{3}{*}{ PCD } & Fluids (Mean \pm SD) & $2.30 \pm 1.25$ & $0.72 \pm 0.27$ & $0.001^{*}$ \\
\hline & Semisolids (Mean \pm SD) & $2.71 \pm 1.78$ & $0.86 \pm 0.25$ & $0.001^{*}$ \\
\hline & $\mathrm{P} 1$ & 0.304 & $0.048^{*}$ & \\
\hline \multirow{3}{*}{ TSD } & Fluids (Mean \pm SD) & $3.39 \pm 1.68$ & $1.11 \pm 0.36$ & $0.001^{*}$ \\
\hline & Semisolids (Mean \pm SD) & $4.36 \pm 2.38$ & $1.24 \pm 0.33$ & $0.001^{*}$ \\
\hline & $\mathrm{P} 1$ & 0.075 & 0.137 & \\
\hline \multirow{3}{*}{ HMED } & Fluids (Mean \pm SD) & $0.39 \pm 0.11$ & $0.36 \pm 0.12$ & $0.291^{*}$ \\
\hline & Semisolids (Mean \pm SD) & $0.50 \pm 0.17$ & $0.45 \pm 0.16$ & 0.239 \\
\hline & $\mathrm{P} 1$ & $0.003^{*}$ & $0.012^{*}$ & \\
\hline
\end{tabular}

Data are expressed as mean and standard deviation.

Student t test was used to compare between both groups

$P$ value $<0.05$ is significant, $P$ value $>0.05$ non-significant.

$\mathrm{P} 1$ represent within group comparison.

$\mathrm{P} 2$ represent between group comparison.

Table 3: Effect of salt taste on temporal measures of swallowing:

\begin{tabular}{cccc}
\hline $\begin{array}{c}\text { Temporal measures of } \\
\text { salt taste in msecs }\end{array}$ & Consistency & Group I (n=30) & Group II (n=30) \\
\hline \multirow{2}{*}{ OTD } & Fluids (Mean \pm SD) & $1.03 \pm 0.45$ & $0.46 \pm 0.17$ \\
& Semisolids (Mean \pm SD) & $1.60 \pm 0.82$ & $0.62 \pm 0.25$ \\
P1 & $0.017^{*}$ & $0.004^{*}$ & $0.03 \pm 0.22$ \\
PTD & Fluids (Mean \pm SD) & $1.47 \pm 0.87$ & $0.60 \pm 0.34$ \\
& Semisolids (Mean \pm SD) & $2.68 \pm 2.92$ & $0.026^{*}$
\end{tabular}




$\begin{array}{ccccc} & \text { Fluids (Mean } \pm \text { SD) } & 1.72 \pm 0.67 & 0.75 \pm 0.19 & 0.001^{*} \\ \text { OCD } & \text { Semisolids (Mean } \pm \text { SD) } & 2.56 \pm 1.52 & 0.90 \pm 0.32 & 0.001^{*} \\ & \text { P1 } & 0.007^{*} & 0.034^{*} & \\ \text { PCD } & 2.23 \pm 1.01 & 0.72 \pm 0.23 & 0.001^{*} \\ & \text { Fluids (Mean } \pm \text { SD) } & 3.49 \pm 2.85 & 0.97 \pm 0.30 & 0.001^{*} \\ \text { TSD } & \text { Semisolids (Mean } \pm \text { SD) } & 0.027^{*} & 0.001^{*} & \\ & \text { P1 } & 3.30 \pm 1.14 & 1.19 \pm 0.30 & 0.001^{*} \\ & \text { Fluids (Mean } \pm \text { SD) } & 4.94 \pm 3.42 & 1.58 \pm 0.30 & 0.001^{*} \\ \text { HMED } & \text { Semisolids (Mean } \pm \text { SD) } & 0.015^{*} & 0.001^{*} & \\ & \text { P1 } & 0.41 \pm 0.09 & 0.42 \pm 0.14 & 0.924 \\ & \text { Fluids (Mean } \pm \text { SD) } & 0.49 \pm 0.15 & 0.57 \pm 0.21 & 0.100\end{array}$

Data are expressed as mean and standard deviation.

Student $\mathrm{t}$ test was used to compare between both groups

$P$ value $<0.05$ is significant, $P$ value $>0.05$ non-significant.

P1 represent within group comparison.

P2 represent between group comparison.

Table 4: Effect of bitter taste in fluids and spicy taste in semisolids on temporal measures of swallowing:

\begin{tabular}{|c|c|c|c|c|}
\hline $\begin{array}{l}\text { Temporal measures } \\
\text { of bitter and spicy } \\
\text { taste in msecs }\end{array}$ & Consistency & Group I $(n=30)$ & Group II $(\mathrm{n}=30)$ & $\mathrm{P} 2$ \\
\hline \multirow{3}{*}{ OTD } & Fluids (Mean \pm SD) & $1.35 \pm 1.05$ & $0.42 \pm 0.14$ & $0.001^{*}$ \\
\hline & Semisolids (Mean \pm SD) & $1.99 \pm 0.82$ & $0.53 \pm 0.16$ & $0.001^{*}$ \\
\hline & $\mathrm{P} 1$ & $0.001^{*}$ & $0.003^{*}$ & \\
\hline \multirow{3}{*}{ PTD } & Fluids (Mean \pm SD) & $2.02 \pm 1.70$ & $0.47 \pm 0.19$ & $0.001^{*}$ \\
\hline & Semisolids (Mean \pm SD) & $2.58 \pm 2.29$ & $0.53 \pm 0.26$ & $0.001^{*}$ \\
\hline & $\mathrm{P} 1$ & $0.039^{*}$ & 0.301 & \\
\hline \multirow{3}{*}{ OCD } & Fluids (Mean \pm SD) & $2.38 \pm 1.87$ & $0.65 \pm 0.19$ & $0.001^{*}$ \\
\hline & Semisolids (Mean \pm SD) & $3.17 \pm 1.47$ & $0.81 \pm 0.22$ & $0.001^{*}$ \\
\hline & $\mathrm{P} 1$ & $0.001^{*}$ & $0.003^{*}$ & \\
\hline \multirow{3}{*}{ PCD } & Fluids (Mean \pm SD) & $2.70 \pm 1.74$ & $0.75 \pm 0.16$ & $0.001^{*}$ \\
\hline & Semisolids (Mean \pm SD) & $3.20 \pm 2.19$ & $0.98 \pm 0.27$ & $0.001^{*}$ \\
\hline & $\mathrm{P} 1$ & $0.015^{*}$ & $0.001^{*}$ & \\
\hline \multirow{3}{*}{ TSD } & Fluids (Mean \pm SD) & $3.99 \pm 2.76$ & $1.16 \pm 0.28$ & $0.001^{*}$ \\
\hline & Semisolids (Mean \pm SD) & $5.14 \pm 2.77$ & $1.51 \pm 0.32$ & $0.001^{*}$ \\
\hline & $\mathrm{P} 1$ & $0.001^{*}$ & $0.001^{*}$ & \\
\hline \multirow{3}{*}{ HMED } & Fluids (Mean \pm SD) & $0.40 \pm 0.15$ & $0.38 \pm 0.13$ & 0.293 \\
\hline & Semisolids (Mean \pm SD) & $0.49 \pm 0.15$ & $0.58 \pm 0.17$ & $0.025^{*}$ \\
\hline & $\mathrm{P} 1$ & $0.001^{*}$ & $0.001^{*}$ & \\
\hline
\end{tabular}

Data are expressed as mean and standard deviation.

Student $\mathrm{t}$ test was used to compare between both groups

$P$ value $<0.05$ is significant, $P$ value $>0.05$ non-significant.

$\mathrm{P} 1$ represent within group comparison.

P2 represent between group comparison. 
3.2.2. Comparison between different bolus tastes as regards their effects on temporal measures of swallowing in the patient group:

\section{A) In liquid bolus:}

Using the ANOVA test, statistically significant differences were found between different tastes of fluids regarding their effects on OTD and OCD, whereas the other temporal measures were not statistically different. Sweet taste showed the shortest OTD, OCD, PCD, TSD, and HMED, whereas bitter taste showed the longest OTD, PTD, OCD, PCD, and TSD compared to other tastes.

Post hoc test analysis revealed a significant difference between sweet and bitter tastes as OTD and OCD were significantly longer in the bitter taste (Tables 5, 6).

\section{B) In semisolid bolus:}

Non-significant differences were found between different tastes of semisolids as regards their effects on temporal measures of swallowing (Table 7).

\subsubsection{Effects of individual bolus tastes on penetration aspiration scale scores:}

In the patient group, PAS scores for all bolus tastes were significantly higher in fluid boluses than in semisolid boluses. When comparing both groups, fluid boluses showed significantly higher PAS scores in the patient group, whatever the bolus taste (Table 8).
3.2.4. Comparison between different bolus tastes as regards their effects on penetration aspiration scale of swallowing in the patient group:

Non-significant differences were found between different tastes as regards their effects on PAS scores (Table 9).

\subsection{Results of FEES:}

\subsubsection{Effects of individual bolus tastes on Mansoura Fees Residue Rating scale (MFRRS):}

In all bolus tastes, both fluid and semisolid consistencies demonstrated greater residue in both vallecula and pyriform in the patient group than in control group, with statistically significant differences for semisolids. Residue scale was predominantly higher in the semisolid consistency than in fluid consistency; however, this difference did not reach significance in either group (Table 10).

\subsubsection{Comparison between different bolus tastes as regards their effects on residue scale of swallowing in the patient group:}

Non-significant differences were found between different tastes as regards their effects on residue scale scores (Table 11).

Table 5: Comparison between different tastes of fluids in patient group as regard their effect on temporal measures:

\begin{tabular}{ccccccc}
\hline $\begin{array}{c}\text { Temporal measures } \\
\text { of fluids in msecs }\end{array}$ & $\begin{array}{c}\text { Sweet } \\
(\text { Mean } \pm \text { SD) }\end{array}$ & $\begin{array}{c}\text { Sour } \\
(\text { Mean } \pm \text { SD) }\end{array}$ & $\begin{array}{c}\text { Salt } \\
(\text { Mean } \pm \text { SD) }\end{array}$ & $\begin{array}{c}\text { Bitter } \\
(\text { Mean } \pm \text { SD) }\end{array}$ & ANOVA (F) & P-value \\
\hline OTD & $0.95 \pm 0.46$ & $1.10 \pm 0.56$ & $1.03 \pm 0.45$ & $1.35 \pm 1.05$ & 2.251 & $0.046^{*}$ \\
PTD & $1.61 \pm 1.04$ & $1.75 \pm 1.39$ & $1.47 \pm 0.87$ & $2.02 \pm 1.70$ & 0.983 & 0.403 \\
OCD & $1.70 \pm 0.75$ & $1.81 \pm 1.07$ & $1.72 \pm 0.67$ & $2.38 \pm 1.87$ & 2.181 & $0.044^{*}$ \\
PCD & $2.12 \pm 0.92$ & $2.30 \pm 1.25$ & $2.23 \pm 1.01$ & $2.70 \pm 1.74$ & 1.216 & 0.307 \\
TSD & $3.07 \pm 1.03$ & $3.39 \pm 1.68$ & $3.30 \pm 1.14$ & $3.99 \pm 2.76$ & 1.413 & 0.243 \\
HMED & $0.35 \pm 0.09$ & $0.39 \pm 0.11$ & $0.41 \pm 0.09$ & $0.40 \pm 0.15$ & 1.747 & 0.161 \\
\hline
\end{tabular}

$\mathrm{F}=$ ANOVA test $*$ significant at the 0.05 level

Table 6: Post hoc test for different tastes of fluids in patient group:

\begin{tabular}{|c|c|c|c|c|c|c|c|c|c|c|c|c|}
\hline \multirow{2}{*}{$\begin{array}{l}\text { Temporal } \\
\text { measures }\end{array}$} & \multicolumn{2}{|c|}{$\begin{array}{c}\text { Sweet vs } \\
\text { Sour }\end{array}$} & \multicolumn{2}{|c|}{$\begin{array}{c}\text { Sweet vs } \\
\text { Salt }\end{array}$} & \multicolumn{2}{|c|}{$\begin{array}{l}\text { Sweet vs } \\
\text { Bitter }\end{array}$} & \multicolumn{2}{|c|}{$\begin{array}{l}\text { Sour vs } \\
\text { Salt }\end{array}$} & \multicolumn{2}{|c|}{$\begin{array}{c}\text { Sour vs } \\
\text { Bitter }\end{array}$} & \multicolumn{2}{|c|}{$\begin{array}{c}\text { Salt vs } \\
\text { Bitter }\end{array}$} \\
\hline & $\mathrm{t}$ & $\mathrm{p}$ & $\mathrm{t}$ & $\mathrm{p}$ & $\mathrm{t}$ & $\mathrm{p}$ & $\mathrm{t}$ & $\mathrm{p}$ & $\mathrm{t}$ & $\mathrm{p}$ & $\mathrm{t}$ & $\mathrm{p}$ \\
\hline OTD & 1.253 & 0.215 & 1.663 & 0.102 & 2.039 & $0.046^{*}$ & 0.568 & 0.572 & 1.167 & 0.248 & 0.681 & 0.499 \\
\hline PTD & 0.654 & 0.516 & 1.567 & 0.123 & 1.129 & 0.264 & 0.944 & 0.349 & 0.467 & 0.643 & 0.544 & 0.588 \\
\hline OCD & 1.433 & 0.157 & 1.823 & 0.073 & 1.838 & $0.030^{*}$ & 0.420 & 0.676 & 0.467 & 0.642 & 0.080 & 0.937 \\
\hline PCD & 1.037 & 0.304 & 1.287 & 0.203 & 1.638 & 0.107 & 0.227 & 0.821 & 0.643 & 0.523 & 0.464 & 0.645 \\
\hline TSD & 1.006 & 0.319 & 1.263 & 0.212 & 1.689 & 0.097 & 0.256 & 0.799 & 0.882 & 0.382 & 0.788 & 0.434 \\
\hline HMED & 0.510 & 0.612 & 0.262 & 0.794 & 1.652 & 0.104 & 0.995 & 0.324 & 1.396 & 0.168 & 2.607 & 0.212 \\
\hline
\end{tabular}

Student $\mathrm{t}$ test $*$ statistically significant at $p<0.05$ 
Table 7: Comparison between different tastes of semisolids as regard their effect on temporal measures in patient group:

\begin{tabular}{|c|c|c|c|c|c|c|}
\hline $\begin{array}{l}\text { Temporal measures } \\
\text { of semisolids } \\
\text { in msecs }\end{array}$ & $\begin{array}{c}\text { Sweet } \\
(\text { Mean } \pm \text { SD })\end{array}$ & $\begin{array}{c}\text { Sour } \\
(\text { Mean } \pm \text { SD) }\end{array}$ & $\begin{array}{c}\text { Salt } \\
(\text { Mean } \pm \text { SD) }\end{array}$ & $\begin{array}{c}\text { Spicy } \\
(\text { Mean } \pm \text { SD) }\end{array}$ & ANOVA (F) & $P$-value \\
\hline OTD & $1.45 \pm 0.80$ & $1.65 \pm 0.86$ & $1.60 \pm 0.82$ & $1.99 \pm 0.82$ & 2.254 & 0.766 \\
\hline PTD & $2.37 \pm 3.12$ & $1.93 \pm 1.70$ & $2.68 \pm 2.92$ & $2.58 \pm 2.29$ & 0.505 & 0.680 \\
\hline OCD & $2.75 \pm 2.33$ & $2.69 \pm 1.73$ & $2.56 \pm 1.52$ & $3.17 \pm 1.47$ & 0.640 & 0.591 \\
\hline PCD & $3.26 \pm 3.24$ & $2.71 \pm 1.78$ & $3.49 \pm 2.85$ & $3.20 \pm 2.19$ & 0.481 & 0.696 \\
\hline TSD & $4.86 \pm 3.89$ & $4.36 \pm 2.38$ & $4.94 \pm 3.42$ & $5.14 \pm 2.77$ & 0.332 & 0.802 \\
\hline HMED & $0.45 \pm 0.14$ & $0.50 \pm 0.17$ & $0.49 \pm 0.15$ & $0.49 \pm 0.15$ & 0.625 & 0.601 \\
\hline
\end{tabular}

$\mathrm{F}=$ ANOVA test $*$ significant at the 0.05 level

Table 8: Effect of bolus taste on PAS scores:

\begin{tabular}{|c|c|c|c|c|}
\hline Taste & Consistency & $\begin{array}{c}\text { Group I PAS } \\
\text { scores }(n=30)\end{array}$ & $\begin{array}{l}\text { Group II PAS } \\
\text { scores }(\mathrm{n}=30)\end{array}$ & P2 \\
\hline \multirow{3}{*}{ Sweet } & Fluids (Mean \pm SD) & $1.20 \pm 0.48$ & $1.00 \pm 0.00$ & $0.027^{*}$ \\
\hline & Semisolids (Mean \pm SD) & $1.00 \pm 0.00$ & $1.00 \pm 0.00$ & - \\
\hline & $\mathrm{P} 1$ & $0.027^{*}$ & - & \\
\hline \multirow{3}{*}{ Sour } & Fluids (Mean \pm SD) & $1.13 \pm 0.35$ & $1.00 \pm 0.00$ & $0.039^{*}$ \\
\hline & Semisolids (Mean \pm SD) & $1.00 \pm 0.00$ & $1.00 \pm 0.00$ & - \\
\hline & $\mathrm{P} 1$ & $0.039^{*}$ & - & \\
\hline \multirow{3}{*}{ Salt } & Fluids (Mean \pm SD) & $1.13 \pm 0.35$ & $1.00 \pm 0.00$ & $0.039^{*}$ \\
\hline & Semisolids $($ Mean \pm SD) & $1.00 \pm 0.00$ & $1.00 \pm 0.00$ & - \\
\hline & $\mathrm{P} 1$ & $0.039^{*}$ & - & \\
\hline \multirow{3}{*}{ Bitter \& Spicy } & Fluids (Mean \pm SD) & $1.13 \pm 0.35$ & $1.00 \pm 0.00$ & $0.039^{*}$ \\
\hline & Semisolids (Mean \pm SD) & $1.00 \pm 0.00$ & $1.10 \pm 0.31$ & 0.078 \\
\hline & P1 & $0.039^{*}$ & 0.078 & \\
\hline
\end{tabular}

Data are expressed as mean and standard deviation.

$P$ value $<0.05$ is significant, $P$ value $>0.05$ non-significant.

Student $\mathrm{t}$ test was used to compare between both groups

P1 represents within group comparison.

$\mathrm{P} 2$ represents between group comparison.

Table 9: Comparison between different tastes of fluids and semisolids in patient group as regard their effect on PAS scores;

\begin{tabular}{|c|c|c|c|c|c|c|c|}
\hline PAS & $\begin{array}{c}\text { Sweet } \\
(\text { Mean } \pm \text { SD })\end{array}$ & $\begin{array}{c}\text { Sour } \\
(\text { Mean } \pm \text { SD })\end{array}$ & $\begin{array}{c}\text { Salt } \\
(\text { Mean } \pm \text { SD })\end{array}$ & $\begin{array}{c}\text { Bitter } \\
(\text { Mean } \pm \text { SD })\end{array}$ & $\begin{array}{c}\text { Spicy } \\
(\mathrm{Mean} \pm \mathrm{SD})\end{array}$ & ANOVA (F) & $P$-value \\
\hline Fluids & $1.20 \pm 0.48$ & $1.13 \pm 0.35$ & $1.13 \pm 0.35$ & $1.13 \pm 0.35$ & & - & - \\
\hline Semisolids & $1.00 \pm 0.00$ & $1.00 \pm 0.00$ & $1.00 \pm 0.00$ & & $1.00 \pm 0.001$ & 3.222 & 0.055 \\
\hline
\end{tabular}

$\mathrm{F}=$ ANOVA test $*$ significant at the 0.05 level

Table 10: Effect of different tastes on residue scale:

\begin{tabular}{|c|c|c|c|c|c|}
\hline Taste & & Consistency & Group I $(n=30)$ & Group II $(n=30)$ & $\mathrm{P} 2$ \\
\hline \multirow{6}{*}{ Sweet } & \multirow{3}{*}{$\mathrm{RP}$} & Fluids & $0.87 \pm 1.20$ & $0.37 \pm 0.49$ & 0.410 \\
\hline & & Semisolids & $1.27 \pm 1.26$ & $0.57 \pm 0.50$ & $0.006^{*}$ \\
\hline & & $\mathrm{P} 1$ & 0.404 & 0.125 & \\
\hline & \multirow{3}{*}{ RV } & Fluids & $0.83 \pm 1.09$ & $0.47 \pm 0.51$ & 0.120 \\
\hline & & Semisolids & $1.93 \pm 1.48$ & $0.40 \pm 0.50$ & $0.001^{*}$ \\
\hline & & $\mathrm{P} 1$ & 0.455 & 0.610 & \\
\hline
\end{tabular}




\begin{tabular}{|c|c|c|c|c|c|}
\hline \multirow{6}{*}{ Sour } & \multirow{3}{*}{$\mathrm{RP}$} & Fluids & $1.17 \pm 1.60$ & $0.57 \pm 0.57$ & 0.058 \\
\hline & & Semisolids & $1.57 \pm 1.19$ & $0.40 \pm 0.56$ & $0.001^{*}$ \\
\hline & & $\mathrm{P} 1$ & 0.277 & 0.259 & \\
\hline & \multirow{3}{*}{ RV } & Fluids & $1.17 \pm 1.12$ & $0.27 \pm 0.45$ & 0.567 \\
\hline & & Semisolids & $2.17 \pm 1.46$ & $0.30 \pm 0.47$ & $0.001^{*}$ \\
\hline & & $\mathrm{P} 1$ & 0.566 & 0.779 & \\
\hline \multirow{6}{*}{ Salt } & \multirow{3}{*}{$\mathrm{RP}$} & Fluids & $0.77 \pm 1.19$ & $0.50 \pm 0.51$ & 0.265 \\
\hline & & Semisolids & $1.13 \pm 1.14$ & $0.43 \pm 0.50$ & $0.003^{*}$ \\
\hline & & $\mathrm{P} 1$ & 0.228 & 0.612 & \\
\hline & \multirow{3}{*}{ RV } & Fluids & $1.03 \pm 0.81$ & $0.47 \pm 0.51$ & 0.323 \\
\hline & & Semisolids & $1.73 \pm 1.48$ & $0.57 \pm 0.50$ & $0.001^{*}$ \\
\hline & & $\mathrm{P} 1$ & 0.270 & 0.447 & \\
\hline \multirow{6}{*}{ Bitter and spicy } & \multirow{3}{*}{$\mathrm{RP}$} & Fluids & $0.97 \pm 1.27$ & $0.53 \pm 0.51$ & 0.165 \\
\hline & & Semisolids & $2.07 \pm 1.51$ & $0.53 \pm 0.57$ & $0.001^{*}$ \\
\hline & & $\mathrm{P} 1$ & 0.233 & 1.000 & \\
\hline & \multirow{3}{*}{ RV } & Fluids & $1.10 \pm 1.24$ & $0.60 \pm 0.50$ & 0.289 \\
\hline & & Semisolids & $1.97 \pm 1.33$ & $0.53 \pm 0.51$ & $0.001^{*}$ \\
\hline & & $\mathrm{P} 1$ & 0.655 & 0.610 & \\
\hline
\end{tabular}

Data are expressed as mean \pm standard deviation.

Student $\mathrm{t}$ test was used to compare between both groups

$P$ value $<0.05$ is significant, $P$ value $>0.05$ non-significant.

$\mathrm{P} 1$ represent within group comparison.

$\mathrm{P} 2$ represent between group comparisons

$\mathrm{RP}=$ Residue in pyriform $\mathrm{RV}=$ Residue in vallecula

Table 11: Comparison between different tastes of fluids and semisolids in patient group as regard their effect on Residue Scale:

\begin{tabular}{|c|c|c|c|c|c|c|c|c|}
\hline & & $\begin{array}{c}\text { Sweet } \\
(\mathrm{Mean} \pm \mathrm{SD})\end{array}$ & $\begin{array}{c}\text { Sour } \\
(\text { Mean } \pm \text { SD) }\end{array}$ & $\begin{array}{c}\text { Salt } \\
(\text { Mean } \pm \text { SD })\end{array}$ & $\begin{array}{c}\text { Bitter } \\
(\mathrm{Mean} \pm \mathrm{SD})\end{array}$ & $\begin{array}{c}\text { Spicy } \\
(\mathrm{Mean} \pm \mathrm{SD})\end{array}$ & ANOVA (F) & P-value \\
\hline \multirow{2}{*}{ Fluids } & $\mathrm{RP}$ & $0.87 \pm 1.20$ & $1.17 \pm 1.60$ & $0.77 \pm 1.19$ & $0.97 \pm 1.50$ & & 0.457 & 0.713 \\
\hline & RV & $0.83 \pm 1.09$ & $1.17 \pm 1.12$ & $1.03 \pm 0.81$ & $1.10 \pm 1.24$ & & 0.539 & 0.657 \\
\hline \multirow{2}{*}{ Semisolids } & $\mathrm{RP}$ & $1.27 \pm 1.26$ & $1.57 \pm 1.19$ & $1.13 \pm 1.14$ & & $2.07 \pm 1.51$ & 3.130 & 0.281 \\
\hline & RV & $1.93 \pm 1.48$ & $2.17 \pm 1.46$ & $1.73 \pm 1.48$ & & $1.87 \pm 1.33$ & 0.474 & 0.701 \\
\hline
\end{tabular}

$\mathrm{F}=\mathrm{ANOVA}$ test $*$ significant at the 0.05 level

\section{DISCUSSION}

The most common and perhaps most crucial cause of neurogenic dysphagia is stroke $\mathrm{e}^{[21]}$. Dysphagia occurs in $37 \%$ to $45 \%$ of stroke patients using screening tests and $64 \%$ to $78 \%$ using instrumental tests ${ }^{[22]}$.

The present study aimed to assess the effect of bolus taste variability on oropharyngeal swallowing in normal individuals versus stroke patients to explore its role in managing oropharyngeal dysphagia. The study was conducted on 60 adults ( 37 males and 23 females) in the age range $30-75$ years, including 30 patients with oropharyngeal dysphagia due to stroke and 30 healthy volunteers.

It has been suggested that bolus volume significantly affects some swallowing measures as upper esophageal sphincter opening duration, laryngeal closure duration, the laryngeal closure-to-upper esophageal sphincter opening interval, and the pharyngeal transit time interval $^{[23]}$. To maintain the reliability of results in this study, fixed volume boluses $5 \mathrm{ml}$ was used in all assessments.

In the present study, most studied patients $(46.7 \%)$ had left cortical stroke. Devroey et al. ${ }^{[24]}$ stated that brain stem strokes are less frequent than cortical lesions and result in the most considerable swallowing compromise.

The videofluoroscopic swallow study assessment can determine the presence, mechanism, and extent of aspiration ${ }^{[25]}$. The most widely used validated scoring system to assess the presence and severity of aspiration and penetration concerning swallowing is the Penetration-Aspiration Scale (PAS) ${ }^{[26]}$. Video records can be analyzed for temporal measures of swallowing. 
VFSS recording of temporal oral and pharyngeal swallowing measures in this study revealed significant prolongation in stroke patients compared to healthy control subjects except for maximal hyoid elevation duration. A recent meta-analysis revealed an association between a longer pharyngeal response time and upper esophageal opening duration and laryngeal aspiration/penetration in dysphagia patients. Nevertheless, there were no studies that related temporal measures and residue ${ }^{[27]}$. Assessment of post-stroke patients by videofluoroscopy detected a significant association between unsafe swallow and a delay in laryngeal vestibule closure and weak tongue propulsion forces ${ }^{[28]}$.

Stroke patients in the current study exhibited significant prolongation of all the semisolid studied temporal measures compared to the fluid consistencies. However, the results of sweet taste fluid and semisolid boluses were comparable. In line with these findings, Abou-Elsaad ${ }^{[29]}$ stated that oral transit time (OTT), pharyngeal transit time (PTT), and total swallow duration (TSD) were significantly shorter for thin liquids than thick liquids and semisolid consistencies. Abou-Elsaad ${ }^{[29]}$ and Steele et al. ${ }^{[30]}$ declared that thin liquids were unsafe and might better be avoided in the early stages of the restoration of oral feeding in stroke patients as they flow quickly with no enough time for the patient to close the airway.

The present results showed that sweet taste liquid boluses improved the oral phase of swallowing in stroke patients with a significant reduction in oral transit and clearance duration compared to bitter taste. Otherwise, adding different flavors to fluid boluses did not induce a significant improvement in the pharyngeal transit and clearance or in the total swallowing time or the duration of maximal hyoid elevation. The oral component of swallowing is voluntary and depends on the lips, teeth, muscles of mastication, and tongue. It is subdivided into the oral preparatory stage and the propulsive stage. Sensory information coming from food and saliva is essential to start and modulate swallowing. Kajii et al. ${ }^{[31]}$ has postulated that peripheral sensory receptors in the taste buds must be stimulated to approximate the sensory threshold of swallows in the brainstem for triggering stronger timely swallows. This might account for the observed favorable decrease in oral transit and clearance duration with sweet liquids in the studied stroke patients.

In the current study, bitter liquid boluses showed the longest temporal measures in stroke patients. Such finding coincides with Leow's study who found that bitter-tasting samples caused the most prolonged oral preparation and floor of mouth contraction time. The sweet and sour tasting samples caused the shortest oral preparation time and the shortest floor of mouth contraction time during swallowing ${ }^{[32]}$. On the contrary, Alves et al. ${ }^{[33]}$ indicated that a bitter bolus did not differ in the duration of oral, pharyngeal, and oesophageal phases of swallowing when compared with a sweet bolus.

In the present study, both sour and salt liquid boluses showed shorter temporal measures than bitter liquid boluses, with the salt boluses being shorter than sour boluses in nearly all temporal measures.

The absence of significant effects of changing taste on most temporal measures, residue scale, and penetration aspiration scale in the studied stroke patients might be due to hypogeusia or quantitative taste disorders caused by central lesions involving taste pathways ${ }^{[34]}$. Alternatively, patients might perceive bolus taste, but the effect of taste did not cause effective oral or pharyngeal motor response due to efferent motor response impairment.

Studies have shown inconsistent results regarding the effects of some taste stimuli in initiating swallowing. Shingai et al. ${ }^{[35]}$ demonstrated an influential role for sour and/or sweet taste stimuli, whereas Yahagi et al. ${ }^{[36]}$ demonstrated improved performance of voluntary swallowing with a salt taste stimulus. Recently, Wang et $a l .{ }^{[37]}$ noted recovery of swallow function in stroke patients with dysphagia in the capsaicin intervention group versus placebo.

The effects of the sour bolus on swallowing showed varying results across studies. Antonious ${ }^{[38]}$ detected non-significant effects of sour taste liquid boluses on pharyngeal transit time in ischemic cerebral vascular accident patients. Hamdy et al. ${ }^{[39]}$ found a slower swallow speed in patients with stroke after the addition of lemon to water. On the other hand, Logemann et al. ${ }^{[40]}$ reported significant improvement in videofluorographic parameters in patients with neurogenic dysphagia by using lemon bolus. Likewise, a high citric acid stimulus significantly improved swallowing and reduced aspiration and penetration in patients with neurogenic oropharyngeal dysphagia ${ }^{[41]}$.

Xiao-ming et al..$^{[42]}$ highlighted the value of sour taste combined with neuromuscular electric stimulation in improving swallowing coordination in patients with acute and subacute stroke. The sour-cold stimulus has also induced improvement of the pharyngeal transit time and has been suggested as a therapeutic aid in stroke patients ${ }^{[11]}$.

A trial of a high concentration sweet-sour and sour flavoured barium sulfate of liquid boluses 
versus unflavored barium sulfate in distilled water in individuals with sensory-based dysphagia showed improved oropharyngeal swallowing, especially with sweet-sour ${ }^{[43]}$. It seems that the effects of bolus taste modification to increase patient's awareness of the presence of bolus in the mouth are still contradictory, with no substantial evidence to support or omit $i^{[44]}$.

The current study revealed significantly higher PAS scores in stroke patients compared to healthy subjects. Additionally, these patients displayed significantly higher severity of aspiration of fluid boluses than semisolids, whatever their taste. As reported by Park et al..$^{[45]}$, several impairments in stroke patients favor the risk of aspiration. These involve leakage of food from the oral cavity, diminished contraction of pharyngeal muscles, residue in the valleculae and the pyriform sinus, impairments in the larynx mobility, and abnormal upper esophageal opening.

Fiberoptic endoscopic evaluation of swallowing provides useful qualitative information on morphology, presence of secretions and residue, presence of penetration and aspiration, the timing of the swallow onset, and clearance of residue ${ }^{[46]}$. Fattori et al. ${ }^{[47]}$ stated that FEES better evaluates post swallow residue because the videoendoscopic method permits a direct view of the hypopharyngeal region. So, even negligible residues can be visible and quickly graded.

The pharyngeal residue is frequently associated with tracheal aspiration in post-stroke patients. Therefore, an accurate description of its severity is a fundamental but difficult challenge ${ }^{[48]}$. Assessment of vallecula and pyriform sinus residue by FEES based on Mansoura FEES Residue Rating Scale (MFRRS) revealed greater residue of both sweet fluids and semisolid boluses in the patient group compared to the control group. The presence of pharyngeal residues in stroke patients is related to the impairment of various biomechanical oropharyngeal swallowing actions, including oral propulsion, pharyngeal contraction, and the opening of the pharyngoesophageal transition ${ }^{[49]}$.

In agreement with the present findings, Onofri et $a l .{ }^{[49]}$ and Alves et al. ${ }^{[50]}$ stated that both stroke and healthy subjects displayed non-significant effects of bolus consistency or taste on the severity of pharyngeal residue scale. However, the residue was predominantly higher in semisolid consistency. On the contrary, Asselin and Dietsch ${ }^{[51]}$ declared that their studied traumatic injured patients showed consistently lower vallecular and pyriform residue with swallowing a sweet-sour bolus.

The present study highlights the shortening effect of sweet liquid bolus on oral transit and clearance duration in stroke patients with oropharyngeal dysphagia. However, the effect of sweet taste on penetration/aspiration and residue has not been proven.

\section{Clinical implication:}

The presentation of various bolus tastes should be a fundamental part of the swallowing evaluation protocol. These evaluations will aid in selecting an

appropriate taste option for the patient. The biomechanical measures of swallowing described in this study may be used to evaluate dysphagia in various pathological disorders, in following the progress of patients with dysphagia, and in evaluating the effects of appropriate therapies.

The results of the current study aid in choosing an appropriate nutritional option for the dysphagic patients. For example, in cases with prolonged oral and pharyngeal transit times, we can use sweet taste (also sour and salt tastes may be used) to improve the onset of oral swallow and pharyngeal transit times as they induced shorter temporal measures.

In other physiological breakdowns, when we need to increase swallowing time to give more time for triggering the swallow reflex, we can use spicy taste as it had the longest temporal measures. The spicy tasted food can be applied in some cultures which prefer this taste like Upper Egypt or India. This necessitates further research.

\section{CONCLUSION}

Different tastes have different temporal measures, with the sweet taste being the shortest and bitter taste being the most prolonged durations in both normal and stroke patients. Neither studied taste correlated significantly with penetration /aspiration of food boluses nor with their residue scale. The food taste management option could be used in different physiological breakdowns, which necessitates further research.

\section{LIMITATIONS OF THE STUDY}

One of the limitations of the current study is the lack of evaluation of neutral taste compared to the other four tastes, which might strengthen the study results.

\section{CONFLICT OF INTEREST}

There are no conflicts of interest. 


\section{FUNDING}

This is an academic research done at Mansoura University hospitals, that did not receive any specific grant from fee-for-service and health care providers.

\section{REFERENCES}

1. Cohen DL, Roffe C, Beavan J, Blackett B, Fairfield CA, Hamdy S, Havard D, McFarlane M, McLauglin C, Randall M, Robson K, Scutt P, Smith C, Smithard D, Sprigg N, Warusevitane A, Watkins C, Woodhouse L, Bath PM. Post-stroke dysphagia: A review and design considerations for future trials. Int J Stroke. 2016;11(4):399-411.

2. Marin S, Serra-Prat M, Ortega O, Clavé P. Cost of oropharyngeal dysphagia after stroke: protocol for a systematic review. BMJ Open. 2018;8(12):e022775.

3. Armstrong JR, Mosher BD. Aspiration pneumonia after stroke: intervention and prevention. The Neurohospitalist. 2011;1(2):85-93.

4. Silva GS. A Taste for Stroke. Rev Neurocienc. 2014;22(1):7.

5. Pauloski BR, Nasir SM. Orosensory contributions to dysphagia: a link between perception of sweet and sour taste and pharyngeal delay time. Physiological reports. 2016;4(11):e12752.

6. Heckmann JG, Stössel C, Lang CJ, Neundörfer B, Tomandl B, Hummel T. Taste disorders in acute stroke: a prospective observational study on taste disorders in 102 stroke patients. Stroke. 2005;36(8): 1690-1694.

7. Baroni AFFB, Fábio SRC, Dantas RO. Fatores de risco para disfunção da deglutição em pacientes com acidente vascular encefálico. Arquivos de Gastroenterologia. 2012;49:118-124.

8. Ferri CP, Schoenborn C, Kalra L, Acosta D, Guerra M, Huang Y, Jacob KS, Llibre Rodriguez JJ, Salas A, Sosa AL, Williams JD, Liu Z, Moriyama T, Valhuerdi A, Prince MJ. Prevalence of stroke and related burden among older people living in Latin America, India and China. J Neurol Neurosurg Psychiatry. 2011;82(10): 1074-1082.

9. Lee KL, Kim DY, Kim WH, Kim EJ, Lee WS, Hahn SJ, Kang MS, Ahn SY. The influence of sour taste on dysphagia in brain injury: blind study. Ann Rehabil Med. 2012;36(3):365-370.

10. Pelletier CA, Lawless HT. Effect of citric acid and citric acid-sucrose mixtures on swallowing in neurogenic oropharyngeal dysphagia. Dysphagia 2003;18(4):231-241.

11. Cola PC, Gatto AR, da Silva RG, Spadotto AA, Ribeiro PW, Schelp AO, Carvalho LR, Henry MA. Taste and temperature in swallowing transit time after stroke. Cerebrovasc Dis Extra. 2012;2(1):45-51.

12. Aviv JE. Prospective, randomized outcome study of endoscopy versus modified barium swallow in patients with dysphagia. Laryngoscope. 2000;110(4):563-574.

13. Aviv JE, Sataloff RT, Cohen M, Spitzer J, Ma G, Bhayani R, Close LG. Cost-effectiveness of two types of dysphagia care in head and neck cancer: a preliminary report. Ear Nose Throat J. 2001;80(8):553-558.

14. Langmore SE, Schatz K, Olson N. Endoscopic and videofluoroscopic evaluations of swallowing and aspiration. Ann Otol Rhinol Laryngol. 1991;100(8):678-681.

15. Wu CH, Hsiao TY, Chen JC, Chang YC, Lee SY. Evaluation of swallowing safety with fiberoptic endoscope: comparison with videofluoroscopic technique. Laryngoscope, 1997;107(3):396-401.

16. Rousmans S, Robin O, Dittmar A, Vernet-Maury E. Autonomic nervous system responses associated with primary tastes. Chemical senses. 2000;25(6):709-718.

17. Leow LP, Huckabee ML, Sharma S, Tooley TP. The influence of taste on swallowing apnea, oral preparation time, and duration and amplitude of submental muscle contraction. Chemical senses. 2007;32(2):119-128.

18. Logemann JA. Dysphagia: evaluation and treatment. Folia Phoniatr Logop. 1995;47(3):140-164.

19. Rosenbek JC, Robbins JA, Roecker EB, Coyle JL, Wood JL. A penetration-aspiration scale. Dysphagia. 1996;11(2):93-98.

20. Sabry A, Coyle JL, Abou-Elsaad T. Mansoura FEES Residue Rating Scale: An anatomically based tool; a preliminary study, Folia phoniatrica et Logopaedica, in press.

21. Daniels SK, Ballo LA, Mahoney MC, Foundas AL. Clinical predictors of dysphagia and aspiration risk: outcome measures in acute stroke patients. Arch Phys Med Rehabil. 2000;81(8):1030-1033.

22. Martino R. Oropharyngeal dysphagia: surveying practice patterns of the speech- languagepathologist. Dysphagia. 2011;19:165-176. 
23. Molfenter SM, Steele CM. Variation in temporal measures of swallowing: sex and volume effects. Dysphagia. 2013;28(2):226-233.

24. Devroey D, Van Casteren V, Buntinx F. Registration of stroke through the Belgian sentinel network and factors influencing stroke mortality. Cerebrovasc Dis. 2003;16(3):272-279.

25. Martin-Harris B, Jones B. The videofluorographic swallowing study. Phys Med Rehabil Clin N Am. 2008;19(4):769-85.

26. Rosenbek JC, Robbins JA, Roecker EB, Coyle JL, Wood JL. A penetration-aspiration scale. Dysphagia. 1996;11(2):93-98.

27. Furkim AM, da Silva RG, Vanin G, Martino R. The association between temporal measures of swallowing with penetration and aspiration in patients with dysphagia: A meta-analysis. Neuro Rehabilitation. 2019;44(1):111-129.

28. Vilardell N, Rofes L, Arreola V, Martin A, Muriana D, Palomeras E, Ortega O, Clavé P. Videofluoroscopic assessment of the pathophysiology of chronic poststroke oropharyngeal dysphagia. Neurogastroenterol Motil. 2017;29(10):1-8.

29. Abou-Elsaad T. Effect of diet variables on the biomechanical measures of swallowing in stroke patients. IFOS. 2003;5131(03):00819-7.

30. Steele CM, Alsanei WA, Ayanikalath S, Barbon CE, Chen J, Cichero JA, Coutts K, Dantas RO, Duivestein J, Giosa L, Hanson B, Lam P, Lecko C, Leigh C, Nagy A, Namasivayam AM, Nascimento WV, Odendaal I, Smith $\mathrm{CH}$, Wang $\mathrm{H}$. The influence of food texture and liquid consistency modification on swallowing physiology and function: a systematic review. Dysphagia. 2015;30(1):2-26.

31. Kajii Y, Shingai T, Kitagawa J, Takahashi Y, Taguchi Y, Noda T, Yamada Y. Sour taste stimulation facilitates reflex swallowing from the pharynx and larynx in the rat. Physiol Behav. 2002;77(2-3):321-325.

32. Leow LP, Huckabee ML, Sharma S, Tooley TP. The influence of taste on swallowing apnea, oral preparation time, and duration and amplitude of submental muscle contraction. Chem Senses. 2007;32(2):119-128.

33. Alves LM, Fabio SR, Dantas RO. Effect of bolus taste on the esophageal transit of patients with stroke. Dis Esophagus. 2013;26(3):305-310.
34. Landis BN, Leuchter I, San Millán Ruíz D, Lacroix JS, Landis T. Transient hemiageusia in cerebrovascular lateral pontine lesions. J Neurol Neurosurg Psychiatry. 2006;77(5):680-683.

35. Shingai T, Miyaoka Y, Ikarashi R, Shimada K. Swallowing reflex elicited by water and taste solutions in humans. Am J Physiol. 1989;256(4 Pt 2):R822R826.

36. Yahagi R, Okuda-Akabane K, Fukami H, Matsumoto N, Kitada Y. Facilitation of voluntary swallowing by chemical stimulation of the posterior tongue and pharyngeal region in humans. Neurosci Lett. 2008;448(1):139-142.

37. Wang Z, Wu L, Fang Q, Shen M, Zhang L, Liu X. Effects of capsaicin on swallowing function in stroke patients with dysphagia: A randomized controlled trial. J Stroke Cerebrovasc Dis. 2019;28(6):1744-1751.

38. Antonious C. The effects of a sour bolus on swallowing apnea duration, swallow/respiratory phase relationship, and pharyngeal transit time in individuals with ischemic cerebral vascular accident. (The William Paterson University of New Jersey). 2010;3:94-23.

39. Hamdy S, Jilani S, Price V, Parker C, Hall N, Power M. Modulation of human swallowing behaviour by thermal and chemical stimulation in health and after brain injury. Neurogastroenterol Motil. 2003;15(1):6977.

40. Logemann JA, Pauloski BR, Colangelo L, Lazarus C, Fujiu M, Kahrilas PJ. Effects of a sour bolus on oropharyngeal swallowing measures in patients with neurogenic dysphagia. J Speech Hear Res. 1995;38(3):556-563.

41. Pelletier CA, Lawless HT. Effect of citric acid and citric acid-sucrose mixtures on swallowing in neurogenic oropharyngeal dysphagia. Dysphagia. 2003;18:231-241.

42. Xiao-ming $X$, Duan J, Yang LJ. Effect of Neuromuscular Electrical Stimulation and Sour Taste Therapy on Dysphagia in Oral Phase in Acute Stroke Patients. Chinese Journal of Rehabilitation Theory and Practice, 2017;23(2):194-198.

43. Dietsch AM, Dorris HD, Pearson WG Jr, DietrichBurns KE, Solomon NP. Taste Manipulation and Swallowing Mechanics in Trauma-Related SensoryBased Dysphagia. J Speech Lang Hear Res. 2019;62(8):2703-2712. 
44. Easterling, C. Management and Treatment of Patients with Dysphagia. Cur Phys Med Rehabil Rep. 2018;6:213-219.

45. Park CH, Kim DK, Lee YT, Yi Y, Lee JS, Kim K, Park $\mathrm{JH}$, Yoon KJ. Quantitative Analysis of Swallowing Function Between Dysphagia Patients and Healthy Subjects Using High-Resolution Manometry. Ann Rehabil Med. 2017;41(5):776-785.

46. Rommel N, Hamdy S. Oropharyngeal dysphagia: manifestations and diagnosis. Nat Rev Gastroenterol Hepatol. 2016;13(1):49-59.

47. Fattori B, Giusti P, Mancini V, Grosso M, Barillari MR, Bastiani L, Molinaro S, Nacci A. Comparison between videofluoroscopy, fiberoptic endoscopy and scintigraphy for diagnosis of oro-pharyngeal dysphagia. Acta Otorhinolaryngol Ital. 2016;36(5): 395-402.
48. Neubauer PD, Hersey DP, Leder SB. Pharyngeal Residue Severity Rating Scales Based on Fiberoptic Endoscopic Evaluation of Swallowing: A Systematic Review. Dysphagia. 2016;31(3):352-9.

49. Onofri SM, Cola PC, Berti LC, da Silva RG, Dantas RO. Correlation between laryngeal sensitivity and penetration/aspiration after stroke. Dysphagia. 2014;29(2):256-61.

50. Alves LM, Secaf M, Dantas RO. Effect of a bitter bolus on oral, pharyngeal and esophageal transit of healthy subjects. Arq Gastroenterol. 2013;50(1):31-34.

51. Asselin M, Dietsch AM. Effect of Taste Stimuli on Swallowing Function in Persons with Traumatic Injuries. Poster Presentation at UNL Summer Research Symposium, 2016 (Aug), Lincoln, NE. 
Appendix (A)

Penetration aspiration scale

(Rosenbek et al., 1996)

1. Material does not enter airway.

2. Material enters the airway, remains above the vocal folds, and is ejected from the airway.

3. Material enters the airway, remains above the vocal folds, and is not ejected from the airway.

4. Material enters the airway, contacts the vocal folds, and is ejected from the airway.

5. Material enters the airway, contacts the vocal folds, and is not ejected from the airway.

6. Material enters the airway, passes below the vocal folds, and is ejected into the larynx or out of the airway.

7. Material enters the airway, passes below the vocal folds, and is not ejected from the trachea despite effort.

8. Material enters the airway, passes below the vocal folds, and no effort is made to eject.

\section{Appendix (B)}

Mansoura FEES Residue Rating Scale (MFRRS)

(Sabry, Coyle, Abou-Elsaad, in Press)

I. Vallecular residue

0: none Right Left Bilateral

2: Shallow/low pooling Right Left Bilateral Midline

3: Moderate pooling Right Left Bilateral

4: Deep/high pooling

5: Overflowing pooling

6: Penetrating pooling

II. Pyriform sinuses residue

0: none Right Left Bilateral

1: Trace Right Left Bilateral

2: Shallow/low pooling Right Left Bilateral

3: Moderate pooling Right Left Bilateral

4: Deep/high pooling

5: Overflowing pooling

6: Penetrating pooling 